\title{
Hinging Hyperplanes Crude Oil Mixing Model for Production Planning Optimization - Supporting Information
}

\author{
Fupei Li, ${ }^{\dagger}$ Feng Qian, ${ }^{* \dagger}$ Chen Fan, ${ }^{\dagger}$ and Vladimir Mahalec ${ }^{*, \ddagger}$ \\ $\dagger$ Key Laboratory of Advanced Control and Optimization for Chemical Processes, Ministry \\ of Education, East China University of Science and Technology, Shanghai 200237, China \\ $\ddagger$ Department of Chemical Engineering, McMaster University, Hamilton, ON, L8S 4L8, \\ Canada \\ E-mail: fqian@ecust.edu.cn; mahalec@mcmaster.ca
}

\section{S-1 Calculation based on pseudo-component method}

A conventional procedure with 4 steps is given by Ref. 1 to develop pseudo-components:

1. Convert ASTM curve into TBP curve if TBP curve is not available.

2. Cut the entire boiling range into a number of cut-point ranges which are used to define pseudo-components.

3. Estimate the density distribution of pseudo-components if only the bulk density is available.

4. Estimate the molecular weight distribution of the entire boiling range if not available and required properties for modeling purpose. 
Since the distillation temperatures are used and the TBP curves are given in common crude oil assay, the step 2 is the key in this wok. The normal boiling point (NBP) temperature $\left(T^{\mathrm{NBP}}\right)$ for a pseudo-component $\left(\mathrm{PC}_{i}\right)$ can be calculated by either the arithmetic (Eq. S.1) or ,more precisely, the integral (Eq. S.2) mean temperature over the corresponding interval of fraction distilled, ${ }^{2}$ where $T^{\mathrm{TBP}}(\cdot)$ represents the TBP curve of a crude oil, $L V_{\mathrm{PC}_{i}}^{\mathrm{U}}$ and $L V_{\mathrm{PC}_{i}}^{\mathrm{L}}$ are the upper and lower bounds of the liquid volume fraction of a pseudo-component in a crude oil. The arithmetic mean temperature is used in this work.

$$
\begin{gathered}
T_{\mathrm{PC}_{i}}^{\mathrm{NBP}}=\frac{T^{\mathrm{TBP}}\left(L V_{\mathrm{PC}_{i}}^{\mathrm{U}}\right)+T^{\mathrm{TBP}}\left(L V_{\mathrm{PC}_{i}}^{\mathrm{L}}\right)}{2}, i=1, \ldots, N \\
T_{\mathrm{PC}_{i}}^{\mathrm{NBP}}=\frac{1}{L V_{\mathrm{PC}_{i}}^{\mathrm{U}}-L V_{\mathrm{PC}_{i}}^{\mathrm{L}}} \int_{L V_{\mathrm{PC}_{i}}^{\mathrm{L}}}^{L V_{\mathrm{PC}_{i}}^{\mathrm{U}}} T^{\mathrm{TBP}}(L V) \mathrm{d} L V, \quad i=1, \ldots, N
\end{gathered}
$$

Then the distribution of the pseudo-components is described by the volumetric fraction $V_{\mathrm{PC}_{i}}^{\text {crude }}$ using the length of the interval referring to the liquid volume fraction on the edges of a pseudo-component boiling-point range (Eq. S.3). And the liquid volume fraction can be calculated by linear interpolation (Eq. S.4) on the set of $M$ points of TBP temperatures and liquid volume fractions $\left(T_{j}^{\mathrm{TBP}}, L V_{j}\right)$ on a crude oil TBP curve, and calculated by linear extrapolation if necessary. ${ }^{3}$ The linear interpolation can be altered to another kind of monotonic interpolation which is better in preserving the shape of the curves. ${ }^{4}$

$$
\begin{gathered}
V_{\mathrm{PC}_{i}}^{\text {crude }}=L V_{\mathrm{PC}_{i}}^{\mathrm{U}}-L V_{\mathrm{PC}_{i}}^{\mathrm{L}}, \quad i=1, \ldots, N \\
L V\left(T^{\mathrm{TBP}}\right) \triangleq \text { liner interpolation }\left(T_{j}^{\mathrm{TBP}}, L V_{j}\right), \quad j=1, \ldots, M
\end{gathered}
$$

Once the pseudo-component distributions have been established and the fractions $r_{k}(k=$ $1, \cdots, L)$ of $L$ crude oils have been determined, the TBP temperatures of the mixed crude oil are calculated by implementing the linear interpolation on the set of cumulated volume fractions and normal boiling point temperatures $\left(\sum_{i=1}^{i-1} V_{\mathrm{PC}_{i}}^{m i x}+\frac{1}{2} V_{\mathrm{PC}_{i}}^{m i x}, T_{\mathrm{PC}_{i}}^{\mathrm{NBP}}\right)$, where $V_{\mathrm{PC}_{i}}^{m i x}$ is 
the fractional mixing of a certain pseudo-component in all crude oils (Eq. S.5 and S.6).

$$
\begin{gathered}
T^{\mathrm{TBP}}(L V) \triangleq \text { liner interpolation }\left(\sum_{i=1}^{i-1} V_{\mathrm{PC}_{i}}^{m i x}+\frac{1}{2} V_{\mathrm{PC}_{i}}^{m i x}, T_{\mathrm{PC}_{i}}^{\mathrm{NBP}}\right) \\
V_{\mathrm{PC}_{i}}^{m i x}=\sum_{k=1}^{L} r_{k} \cdot V_{\mathrm{PC}_{i}}^{\text {crude }}
\end{gathered}
$$

\section{S-2 Training the two-layer perceptron}

The two-layer perceptron is built by using Keras $^{5}$ and TensorFlow ${ }^{6}$ backend in Python 3.6. The Keras is a library that provides highly powerful and abstract building blocks to build deep learning networks as well as feedforward neural networks. The building blocks Keras provides are built using TensorFlow for building computational graphs, automatically deriving gradients, etc. Keras supports both CPU and GPU computation and is a great tool for quickly prototyping ideas. ${ }^{5}$ In this paper, the computational environment is a $3.2 \mathrm{GHz}$ Intel Core i7-8700 desktop machine. The the graph of the two-layer perceptron is show in Fig. S1. The THETA layer with ReLU activation functions simulates the $\boldsymbol{\theta}_{i}$. The SIGN layer with linear activation functions simulates the $c_{i}$, also as the output layer.

The regression of crude oil mixing data based on pseudo-component method is carried out by this neural network. The inputs are the crude oil fractions and liquid volume fractions. The outputs are the TBP temperatures. The training data is the normalized TBP temperatures in the range of $[0,1]$ calculated by Eq. S.7. The normalization makes the training input and output in the same scale. And the weights and biases in the neural network are randomly initialize within $(-1,1)$ range via uniform distribution since the $c_{i}$ can take $\{-1,+1\}$. The loss function is the mean square error (MSE) defined by Eq. S.8. The model is evaluated by the mean absolute error (MAE) defined by Eq. S.9. The optimizer is Adam ${ }^{7}$ with default parameters in Keras, which only requires first-order gradients with little

memory requirement for efficient stochastic optimization. The method computes individual 


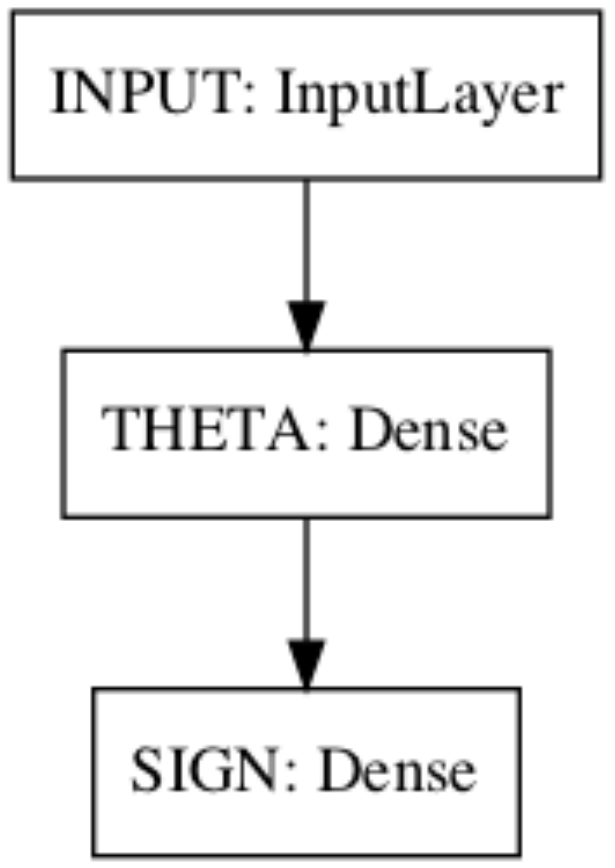

Figure S1: Two-layer perceptron structure

adaptive learning rates for different parameters from estimates of first and second moments of the gradients. ${ }^{7}$ The other parameters in training (e.g. batch size, the number of epoch) are chosen as follow. The batch size is a hyperparameter that defines the number of samples to be processed before updating the internal model parameters, ${ }^{5}$ which is 500 considering the sample size and the computational time. The number of epochs is a hyperparameter that defines the number times that the learning algorithm will work through the entire training dataset. One epoch means that each sample in the training dataset has had an opportunity to update the internal model parameters. An epoch is comprised of one or more batches. The number of epochs is traditionally large, thereby allowing the learning algorithm to run until the error from the model has been sufficiently minimized. ${ }^{5}$ For example, the 500 epochs training of the neural network with 64 nodes is shown in Fig. S2. Therefore, 500 epochs is enough for out test with 8-128 nodes. Then the training is done by the Keras library. After the training the predicted TBP temperatures are calculated by Eq. S.10. The final values of the parameters of weights $\boldsymbol{w}$ and bias $b$ have been calculated through the inverse normalization in the paper. 


$$
\begin{gathered}
y=\frac{T^{\mathrm{TBP}}-T_{m i n}^{\mathrm{TBP}}}{T_{\max }^{\mathrm{TBP}}-T_{m i n}^{\mathrm{TBP}}} \\
M S E=\frac{\sum_{i=1}^{n}\left(y_{i}-\hat{y}_{i}\right)^{2}}{n} \\
M A E=\frac{\sum_{i=1}^{n}\left|y_{i}-\hat{y}_{i}\right|}{n} \\
T^{\mathrm{TBP}}=y \times\left(T_{\max }^{\mathrm{TBP}}-T_{\min }^{\mathrm{TBP}}\right)+T_{m i n}^{\mathrm{TBP}}
\end{gathered}
$$

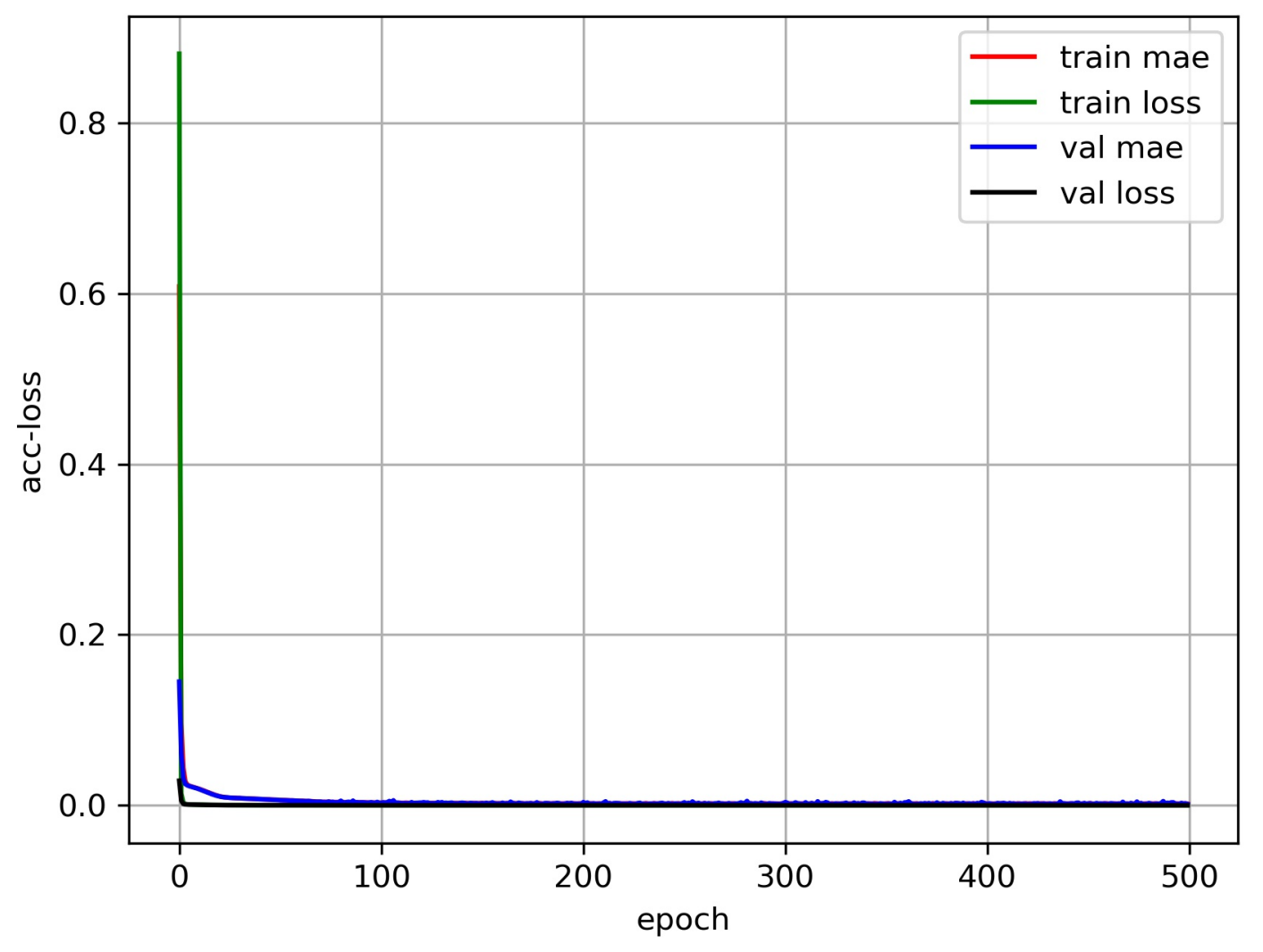

Figure S2: Training epoch 


\section{S-3 Results of crude oil recipes and distillation cut yields}

Table S1: Hinging hyperplanes model (Model 6) results

\begin{tabular}{|c|c|c|c|c|c|c|c|c|c|c|c|c|c|c|}
\hline & \multicolumn{5}{|c|}{ Crude oil } & & \multicolumn{6}{|c|}{ Distillation cut } & \multirow[t]{2}{*}{ Unit } \\
\hline & & $\mathrm{CO} 3$ & $\mathrm{CO} 4$ & CO5 & $\mathrm{CO} 6$ & $\mathrm{CO} 7$ & & $\mathrm{LN}$ & $\mathrm{HN}$ & $\mathrm{K}$ & $\mathrm{D}$ & VGO & VR & \\
\hline \multirow{2}{*}{ Case1 } & $C S_{i}$ & 9094.8 & 0.0 & 5905.2 & 0.0 & 10000.0 & $P D_{j}$ & 1625.2 & 4554.1 & 2289.5 & 5652.7 & 7735.7 & 3142.8 & \\
\hline & $r_{i}$ & 0.3638 & 0.0000 & 0.2362 & 0.0000 & 0.4000 & $y_{j}$ & 0.0650 & 0.1822 & 0.0916 & 0.2261 & 0.3094 & 0.1257 & \\
\hline \multirow{2}{*}{ Case2 } & $C S_{i}$ & 5481.1 & 0.0 & 10000.0 & 0.0 & 9518.9 & $P D_{j}$ & 1576.4 & 4606.2 & 2292.7 & 5702.4 & 7711.6 & 3110.8 & \multirow[t]{2}{*}{ bbl } \\
\hline & $r_{i}$ & 0.2192 & 0.0000 & 0.4000 & 0.0000 & 0.3808 & $y_{j}$ & 0.0631 & 0.1842 & 0.0917 & 0.2281 & 0.3085 & 0.1244 & \\
\hline \multirow{2}{*}{ Case3 } & $C S_{i}$ & 10000.0 & 0.0 & 10000.0 & 0.0 & 5000.0 & $P D_{j}$ & 1561.8 & 4727.8 & 2300.8 & 5735.3 & 7557.6 & 3116.7 & \multirow[t]{2}{*}{ bbl } \\
\hline & $r_{i}$ & 0.4000 & 0.0000 & 0.4000 & 0.0000 & 0.2000 & $y_{j}$ & 0.0625 & 0.1891 & 0.0920 & 0.2294 & 0.3023 & 0.1247 & \\
\hline
\end{tabular}

Table S2: Hinging hyperplanes model inverse function (Model 7) results

\begin{tabular}{|c|c|c|c|c|c|c|c|c|c|c|c|c|c|c|}
\hline & \multicolumn{5}{|c|}{ Crude oil } & & \multicolumn{6}{|c|}{ Distillation cut } & \multirow[t]{2}{*}{ Unit } \\
\hline & & CO3 & $\mathrm{CO} 4$ & CO5 & CO6 & $\mathrm{CO} 7$ & & $\mathrm{LN}$ & $\mathrm{HN}$ & $\mathrm{K}$ & $\mathrm{D}$ & VGO & VR & \\
\hline \multirow{2}{*}{ Case1 } & $C S_{i}$ & 10000.0 & 0.0 & 5000.0 & 0.0 & 10000.0 & $P D_{j}$ & 1628.9 & 4552.0 & 2239.5 & 5679.6 & 7717.6 & 3182.5 & \multirow[t]{2}{*}{$\mathrm{bbl}$} \\
\hline & $r_{i}$ & 0.4000 & 0.0000 & 0.2000 & 0.0000 & 0.4000 & $y_{j}$ & 0.0652 & 0.1821 & 0.0896 & 0.2272 & 0.3087 & 0.1273 & \\
\hline \multirow{2}{*}{ Case2 } & $C S_{i}$ & 5000.0 & 0.0 & 10000.0 & 0.0 & 10000.0 & $P D_{j}$ & 1575.9 & 4576.7 & 2285.1 & 5765.0 & 7672.0 & 3125.3 & \multirow[t]{2}{*}{ bbl } \\
\hline & $r_{i}$ & 0.2000 & 0.0000 & 0.4000 & 0.0000 & 0.4000 & $y_{j}$ & 0.0630 & 0.1831 & 0.0914 & 0.2306 & 0.3069 & 0.1250 & \\
\hline \multirow{2}{*}{ Case3 } & $C S_{i}$ & 10000.0 & 0.0 & 10000.0 & 0.0 & 5000.0 & $P D_{j}$ & 1557.3 & 4710.6 & 2287.6 & 5789.5 & 7535.2 & 3119.8 & \multirow[t]{2}{*}{ bbl } \\
\hline & $r_{i}$ & 0.4000 & 0.0000 & 0.4000 & 0.0000 & 0.2000 & $y_{j}$ & 0.0623 & 0.1884 & 0.0915 & 0.2316 & 0.3014 & 0.1248 & \\
\hline
\end{tabular}

Table S3: Pseudo-component based model (Model 8) results

\begin{tabular}{|c|c|c|c|c|c|c|c|c|c|c|c|c|c|c|}
\hline & \multicolumn{5}{|c|}{ Crude oil } & & \multicolumn{6}{|c|}{ Distillation cut } & \multirow[t]{2}{*}{ Unit } \\
\hline & & CO3 & $\mathrm{CO} 4$ & CO5 & CO6 & $\mathrm{CO} 7$ & & LN & $\mathrm{HN}$ & $\mathrm{K}$ & $\mathrm{D}$ & VGO & VR & \\
\hline \multirow{2}{*}{ Case1 } & $C S_{i}$ & 10000.0 & 0.0 & 5000.0 & 0.0 & 10000.0 & $P D_{j}$ & 1590.8 & 4597.8 & 2221.4 & 5679.7 & 7793.5 & 3116.7 & \multirow[t]{2}{*}{ bbl } \\
\hline & $r_{i}$ & 0.4000 & 0.0000 & 0.2000 & 0.0000 & 0.4000 & $y_{j}$ & 0.0636 & 0.1839 & 0.0889 & 0.2272 & 0.3117 & 0.1247 & \\
\hline \multirow{2}{*}{ Case2 } & $C S_{i}$ & 5000.0 & 0.0 & 10000.0 & 0.0 & 10000.0 & $P D_{j}$ & 1530.2 & 4614.1 & 2251.7 & 5818.4 & 7673.4 & 3112.2 & \multirow[t]{2}{*}{ bbl } \\
\hline & $r_{i}$ & 0.2000 & 0.0000 & 0.4000 & 0.0000 & 0.4000 & $y_{j}$ & 0.0612 & 0.1846 & 0.0901 & 0.2327 & 0.3069 & 0.1245 & \\
\hline \multirow{2}{*}{ Case3 } & $C S_{i}$ & 10000.0 & 0.0 & 10000.0 & 0.0 & 5000.0 & $P D_{j}$ & 1511.1 & 4734.9 & 2295.3 & 5796.4 & 7539.7 & 3122.6 & \multirow[t]{2}{*}{ bbl } \\
\hline & $r_{i}$ & 0.4000 & 0.0000 & 0.4000 & 0.0000 & 0.2000 & $y_{j}$ & 0.0604 & 0.1894 & 0.0918 & 0.2319 & 0.3016 & 0.1249 & \\
\hline
\end{tabular}

\section{References}

(1) Chang, A.-F.; Pashikanti, K.; Liu, Y. A. Refinery Engineering: Integrated Process Modeling and Optimization; John Wiley \& Sons, Ltd, 2013; pp 1-55.

(2) Eckert, E.; Vaněk, T. New approach to the characterisation of petroleum mixtures used in the modelling of separation processes. Computers \& Chemical Engineering 2005, 30, 343-356. 
(3) Fu, G.; Mahalec, V. Comparison of Methods for Computing Crude Distillation Product Properties in Production Planning and Scheduling. Industrial Ef Engineering Chemistry Research 2015, 54, 11371-11382.

(4) Kelly, J. D.; Menezes, B. C.; Grossmann, I. E. Distillation blending and cutpoint temperature optimization using monotonic interpolation. Industrial \& Engineering Chemistry Research 2014, 53, 15146-15156.

(5) Ketkar, N. Deep learning with Python; Springer, 2017; pp 97-111.

(6) Abadi, M. et al. TensorFlow: Large-Scale Machine Learning on Heterogeneous Systems. 2015; https://www.tensorflow.org/, Software available from tensorflow.org.

(7) Kingma, D. P.; Ba, J. Adam: A method for stochastic optimization. arXiv preprint

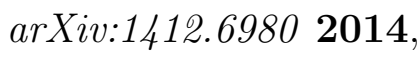

